\title{
Population I Stellar Structure and Evolution: facing the lingering difficulties to make a step forward
}

\author{
André Maeder \\ Geneva Observatory, CH-1290 Sauverny, Switzerland
}

\section{Introduction}

The understanding of stellar structure, evolution and pulsations is a key piece of the proper understanding of the Universe. The interpretation of the luminosity and spectra of galaxies, the properties of star populations and of starbursts, the calibration of distances, the nucleosynthetic prescriptions etc. . are all requiring good models of stellar structure and evolution. The many interesting and new results in the field have been presented in several recent reviews by Maeder (1991), Chiosi et al. (1992), Maeder \& Conti (1994). Table 1 below presents a list of the recent grids of stellar models. The various columns give the author's name, then the mass range, the metallicity $\mathrm{Z}$ range, the kind of convective assumption adopted, where "Schw." means Schwarzschild's criterion, "oversh" means with convective overshooting, "all" means that different assumptions have been used. In the last column, a few remarks are given. It is interesting to note that the models mentioned above often arrive to very different conclusions about the kind of mixing supported by the observations. Authors of models with overshooting generally conclude that overshooting is best supported, authors of models without overshooting favour the absence of overshooting, while authors who consider various convective assumptions generally favour semiconvection (Stothers \& Chin 1991; Mowlawi \& Forestini 1994).

Rather than reviewing all the arguments for and against some specific convective assumptions I shall examine here the various discrepancies between stellar models and observations. Hopefully, from a good appraisal of the existing discrepancies we may learn more about the future improvements needed in model physics, in particular regarding mixing. This is not of minor consequences since all model outputs depend on specific mixing assumptions.

\section{Extended cluster main sequences}

It has been known for a long time that the upper main sequences of clusters in the age range from that of Pleiades to M67 cannot be well fitted with stellar models using Schwarzschild's criterion (cf. Maeder \& Meynet 1989). The observed sequence goes to a larger distance from the ZAMS than predicted. A comparison of the HR diagrams of 30 clusters with isochrones (cf. Meynet et al. 1993) well confirms the need of some core extension to properly account for the upper main sequences of clusters. Some cases, like that of NGC 4651, are beautiful illustrations of the necessity of enlarged cores. For very young clusters, such an effect also seems present (see §7); however the sparse nature of 
the sequence does not allow us to be very affirmative about the top of the main sequence.

The extended cluster sequences have generally been interpreted in terms of convective cores extended by overshooting. An increase of the core by about $0.20 \mathrm{H}_{p}$ is necessary, where $\mathrm{H}_{p}$ is the local pressure height. It is also quite clear that, even with the new OPAL opacities, some core extension is necessary (cf. Meynet et al. 1992). Other features in the HR diagrams of clusters also support the same conclusion (cf. Maeder \& Meynet 1989; Lattanzio et al. 1991; Brocato et al. 1994); however, they are generally less straightforward in interpretation and could be partly attributed to other effects.

Actually one can be rather confident that some small core increase with respect to what is predicted by Schwarzschild's criterion is necessary. However, the origin is certainly uncertain, it could be overshooting, but any additional mixing process in the deep interior would do the same.

\section{3. $\mathrm{N} / \mathrm{C}$ and ${ }^{13} \mathrm{C} /{ }^{12} \mathrm{C}$ enrichments on the Red Giant Branch}

The internal distribution of chemical elements built during MS evolution is reached by the deep external convective zone in the red giant branch and this produces the first dredge-up of chemical elements. The changes of $\mathrm{N} / \mathrm{C}$ as a function of luminosity on the red giant branch have been calculated for stars of different initial masses by Charbonnel (1994). Similar curves are obtained, for example, for the abundance ratio ${ }^{13} \mathrm{C} /{ }^{12} \mathrm{C}$. One notices that in principle, for sufficiently accurate measurements, one would be able to have some mass estimate of red giants in the field.

Various observational comparisons (cf. Sweigart et al. 1989; Gilroy \& Brown 1991; Charbonnel 1994) well support the theoretical results for the first dredgeup: the luminosity and amplitude of the changes in $\mathrm{N} / \mathrm{C}$ and ${ }^{13} \mathrm{C} /{ }^{12} \mathrm{C}$ are correctly predicted. Incidentally, this shows that there is no need of a large overshooting below the convective envelopes as assumed in some models. Also, the results on helioseismology (cf. Gilliland, this meeting) show a good correspondence of the calculated and "observed" lower boundary of the solar convective zone. Comparisons for low-mass stars $\left(\mathrm{M}<2 \mathrm{M}_{\odot}\right)$ on the upper red giant branch show, however, significant discrepancies: N/C and ${ }^{13} \mathrm{C} /{ }^{12} \mathrm{C}$ are higher than predicted by about a factor of 3 (see ref. above). This suggests (cf. Charbonnel 1994) the occurrence of some extra-mixing appearing on the upper red giant branch. Clues are given that the extra-mixing needed to account for the CNO observations is only efficient when the hydrogen-burning shell reaches the chemical discontinuity created by the convective envelope at its maximum extent.

\section{Abundances in Cepheids and AGB stars}

There are not enough data on abundances in Cepheids to allow a correct comparison of observations and models. However, it is interesting to emphasize that the Cepheid models predict different surface He-contents and N/C according to metallicity $\mathrm{Z}$ and masses (cf. Schaller et al. 1992). In general, the higher the mass, the larger the enrichments since convective dredge-up is more efficient. 
Fig. 1 shows the HR diagram for $Z=0.001$ with indications of the Cepheid instability strip and of the regions of slow He-burning for $\mathrm{Z}=0.001$ and $\mathrm{Z}=0.020$. One notices that the intersections of the lines of slow He-burning with the instability strip occur at different $L$, a well known fact which is responsible for the different distributions of luminosities and periods according to the metallicity of the galaxy (cf. Iben \& Tuggle 1975). However, the new point is that the surface He-content $\mathrm{Y}_{s}$ is expected to be much lower at lower $\mathrm{Z}: \mathrm{Y}_{s}=0.243$ at $\mathrm{Z}=0.001$ compared to $Y_{s}=0.33$ at $Z=0.020$. This is rather different from the value $Y_{s}$ $=.29$ found by Iben \& Tuggle for Cepheids at various Z. Simultaneously, the $\mathrm{N} / \mathrm{C}$ ratio should be of the order of 0.7 to 1 in Cepheids compared to a value of 4 for the cosmic abundances. The large helium differences result from the difference of the dredge-up as well as from the initial values. The difference in helium content should have some consequences for the driving mechanism and thus for the amplitudes of Cepheid pulsations at different Z. Such differences of amplitude distributions between the Galaxy, LMC and SMC are known to exist (cf. van Genderen 1978) and we may suspect a relation with the different helium contents.

Table 1. Recent grids of models (only the most recent reference in each series is given)

\begin{tabular}{|c|c|c|c|c|}
\hline GRIDS & $\mathbf{M}$ & $\mathbf{Z}$ & $\operatorname{mix}$. & REMARKS \\
\hline $\begin{array}{c}\text { Brocato \& } \\
\text { Castellani } \\
\quad 1993\end{array}$ & $0.6-20 \mathrm{M}_{\odot}$ & $.002-.02$ & SCHW & $\begin{array}{l}\text { - Laol Opacities } \\
\text { - Concl.: no overshoot }\end{array}$ \\
\hline $\begin{array}{c}\text { Claret \&z } \\
\text { Gimenea } \\
1992\end{array}$ & $1-40 M_{\odot}$ & $.01-.03$ & OVERSH & $\begin{array}{l}\text { - Structure constants } \\
\text { for binaries }\end{array}$ \\
\hline $\begin{array}{c}\text { Geneva } \\
\text { (Meynet et al.) } \\
1994\end{array}$ & $0.8-120 \mathrm{M}_{\odot}$ & $.001-0.04$ & OVERSH & $\begin{array}{l}\text { - Isochrones } \\
\text { - Various } \dot{M} \text {; WR } \\
\text { - Chemistry; pulsation }\end{array}$ \\
\hline $\begin{array}{c}\text { Lattanzio } \\
1991\end{array}$ & $1-5 \mathrm{M}_{\odot}$ & $.001-.02$ & SCHW & $\begin{array}{l}\text { - Laol Opacities } \\
\text { - From ZAMS, ZAHB }\end{array}$ \\
\hline $\begin{array}{c}\text { Mowlawi \& } \\
\text { Forestini } \\
1994\end{array}$ & $2.5-20 \mathrm{M}_{\odot}$ & .02 & ALL & $\begin{array}{l}\text { - Pre-MS evolution } \\
\text { - Concl.: semiconvection }\end{array}$ \\
\hline $\begin{array}{c}\text { Padova } \\
\text { (Bertelli et al.) } \\
1994\end{array}$ & $0.6-120 \mathrm{M}_{\odot}$ & $.0004-.10$ & OVERSH & $\begin{array}{l}\text { - Isochrones } \\
\text { - Population models } \\
\text { - Chemistry }\end{array}$ \\
\hline $\begin{array}{c}\text { Stothers \&z} \\
\text { Chin } \\
1992\end{array}$ & $1.5-60 \mathrm{M}_{\odot}$ & $.02-.03$ & ALL & $\begin{array}{l}\text { - Tests } \\
\text { - Concl.: no or small } \\
\text { overshooting }\end{array}$ \\
\hline $\begin{array}{c}\text { VandenBerg } \\
1992\end{array}$ & $0.15-1.25 \mathrm{M}_{\odot}$ & $.0001-.007$ & SCHW & $\begin{array}{l}\text { - Laol Opacities } \\
\text { - O-enhanced for low Z }\end{array}$ \\
\hline $\begin{array}{c}\text { Vassiliadis \& } \\
\text { Wood } \\
1993\end{array}$ & $0.89-5.0 \mathrm{M}_{\odot}$ & $.001-.016$ & SCHW & $\begin{array}{l}\text { - Laol Opacities } \\
\text { - Through TP - AGB phase }\end{array}$ \\
\hline
\end{tabular}

Recent models of TP-AGB stars by Vassiliadis \& Wood (1993) use a new algorithm for the mass loss rates $\dot{M}$, based on the observed relation between $\dot{M}$ 


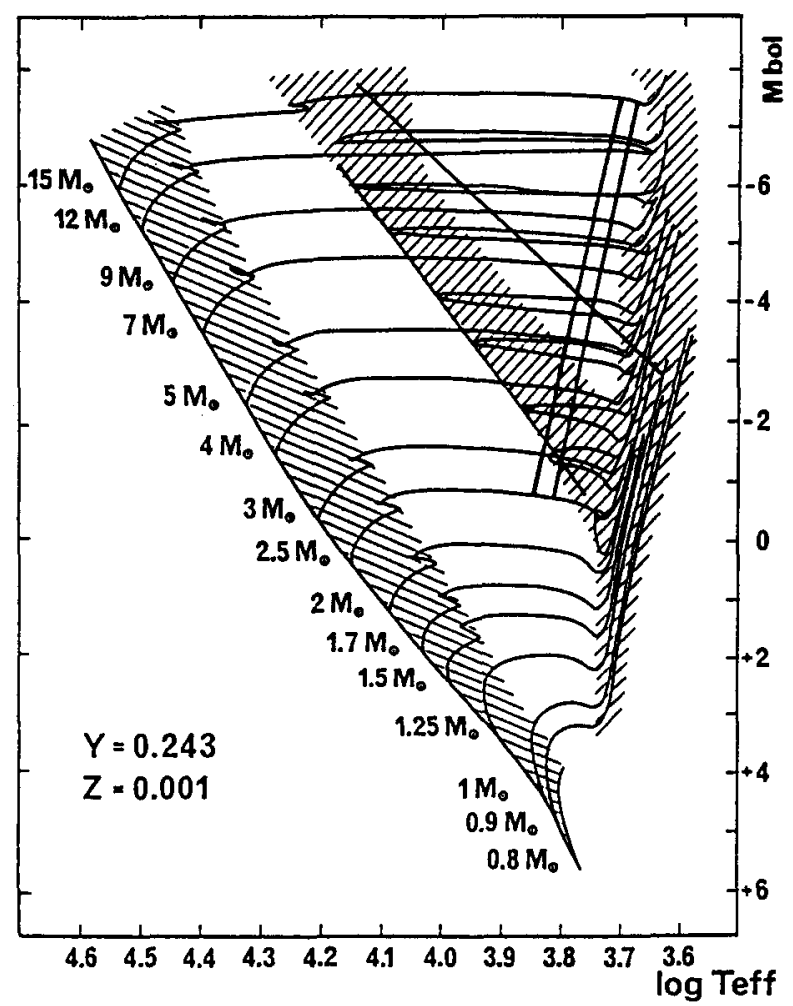

Figure 1. Grids of models at $\mathrm{Z}=0.001$ and $\mathrm{Y}=.234$ (from Schaller et al. 1992) with indication of the Cepheid instability strip. The line connecting the extremity of the blue loops for models with $\mathrm{Z}=0.02$ and $\mathrm{Y}=0.28$ is also given. The surface helium content for stars on the blue loops are very different: $\mathrm{Y}_{s}=0.243$ at $\mathrm{Z}=0.001$ and $\mathrm{Y}_{s}=0.33$ at $\mathrm{Z}=0.02$.

and the pulsation periods of Miras. These models correctly predict the luminosity of the RGB tip as well as the final vs. initial mass relation. However, the long standing difficulty of the models to produce C-stars at a luminosity as low as that observed is still unsolved. Of course, some tricks such as changing the mixing length ratio or $\mathrm{Z}$ may help, but the problem of an earlier efficient transport of $\mathrm{C}$ to the stellar surface remains.

\section{He and mass discrepancy in O-stars}

The problem of rotational mixing has a long history (cf. Tassoul 1978; Zahn 1994). In this context, the recent finding of Herrero et al. (1992) that all fast rotators among $\mathrm{O}$-stars show surface He-enrichments is a very important result. It shows that rotationally induced mixing is strong enough to transport to the surface the newly processed elements during a fraction of the MS lifetimes. 
This implies that all stellar properties are influenced by this effect: lifetimes, luminosities, nucleosynthesis, etc...In $\S 10$ below we try to explain why such effects preferentially occur in the most massive stars. We may also mention the so-called ON-stars. These are O-type stars, often close to the zero-age sequence and which show He- and N-enrichments (Walborn 1988; Howarth \& Prinja 1989). A substantial fraction (about 50\%) of them are binaries and some are also fast rotators. Their location in the HR diagram suggests (cf. Maeder 1987) the possibility of homogeneous evolution as a result of tidal or rotational mixing.

Another discrepancy was also claimed for O-stars: the so-called mass discrepancy (cf. Kudritzki et al. 1992; Herrero et al. 1992). The claimed effects were very large: a $50 \mathrm{M}_{\odot}$ star with a mass value assigned from the luminosity (evolutionary mass) might have a spectroscopic mass of $20 \mathrm{M}_{\odot}$. Doubts were cast on the reality of this discrepancy by Lamers \& Leitherer (1993) and more recently by Schaerer (1995) who showed that the mass discrepancy was mainly due to the fact that the spectroscopic masses were based on atmosphere models, which did not account for the spherical extension of stars. This explains why the discrepancy was found to be larger for supergiants and Of stars than for MS stars. We may also notice that if there is some He-enrichment in O-stars, there should normally be some change in the M-L relation. However, in view of the observed He-enrichments, a $45 \mathrm{M}_{\odot}$ star, say, could possibly shine like a standard model of $50 \mathrm{M}_{\odot}$, but a $20 \mathrm{M}_{\odot}$ star not like one of $50 \mathrm{M}_{\odot}$.

\section{He- and N-excesses in OBA supergiants and in SN $1987 \mathrm{~A}$}

For the time being the most important result in the field is still that by Walborn $(1976,1988)$ who showed that ordinary OB supergiants have He- and Nenrichments as a result of CNO processing, and that it is only the small group of peculiar OBC stars that have the normal cosmic abundance ratios. Several other recent studies fully confirm the above conclusions (cf. Howarth \& Prinja 1989; Herrero et al. 1992; Gies \& Lambert 1992). Also, B-supergiants in the LMC and SMC generally show He- and N-enrichments. A high-dispersion study of LMC B-supergiants by Fitzpatrick \& Bohannan (1993, and reference therein) found among $62 \mathrm{~B} 0.7$ to B3 supergiants only seven OBC stars, while the remainder showed evidence of strongly contaminated surfaces by CNO processing. The progenitor of SN 1987 A, which was a B-supergiant, also had N/C and N/O ratios larger than solar values by factors 37 and 12 respectively (Fransson et al. 1989); however, in this special case the B-supergiant was obviously at a more advanced stage than most B-supergiants on the average. Anyhow, there are enough results to show that the general rule among B-supergiants is $\mathrm{He}$ - and $\mathrm{N}$-enrichments, and this places very severe constraints on the models.

There are two alternatives to explain the He- and $\mathrm{N}$-enrichments in supergiants and both bring about new problems.

I) The first alternative is that the blue supergiants are on the blue loops after a first red supergiant stage, where dredge-up has occurred producing the observed surface enrichments. The problems related to this hypothesis are the following: 1 . At $\mathrm{Z}=0.02$, current grids of models (Schaller et al. 1992; Alongi et al. 1993; Arnett 1991; Brocato \& Castellani 1993) only predict blue loops for 
masses equal or lower than $15 \mathrm{M}_{\odot}$. At higher masses there are no blue loops and no predicted enrichments. 2. The blue loops, when they exist (for $\mathrm{M} \leq$ $15 \mathrm{M}_{\odot}$ ), do not go leftwards enough to reach continuity with the main sequence and this creates the so-called blue Hertzsprung gap (see below). 3. Even on the blue loops the predicted enrichments do not seem high enough to account for the observations. This was shown for three A-supergiants (Venn 1993), for four F-supergiants (Luck \& Lambert 1985) and also in the case of SN 1987 A. II) A possibility is that some blue supergiants are still on the MS. This is not predicted by current stellar models. However, we may assume that the effects producing enriched O-stars (see §5) are also active in lighter lower mass stars and that they lead to surface enrichments in supergiants resulting from a larger range of initial masses.

This case of supergiants shows that we still need to understand their evolutionary stage and that, in any case, additional mixing is necessary to explain their surface abundances.

\section{The blue Hertzsprung gap and the ledge}

As mentioned above, the observed distribution of massive stars in the HR diagram shows a continuous distribution from the MS to the blue and yellow supergiants, with no sign of the predicted gap at the end of the MS (Stothers \& Chin 1977; Chiosi et al. 1978; Meylan \& Maeder 1982; Chiosi et al. 1992). Several different possible explanations have been proposed in terms of enhanced opacities (Nasi \& Forieri 1990), of scatter in mass loss rates (Meynet et al. 1994), extended atmospheres and binaries (Tuchmann \& Wheeler 1989, 1990). Our feeling is that no good solution has been found for this problem yet and that new evolutionary tracks are needed to account for this important fact.

A more or less visible drop-off in the number of LMC supergiants beyond a line somehow parallel to the MS, but shifted by $\sim 0.6$ dex in log $\mathrm{T}_{\text {eff }}$ was claimed by Fitzpatrick \& Garmany (1990) who called it "the ledge". I am not convinced that this is a very constraining effect since most models predict a rather fast evolution of supergiants between $\log \mathrm{T}_{\text {eff }}=3.9$ and 3.6 ; thus the ledge is a rather unclear and not constraining feature.

\section{8. $\mathbf{B} / \mathbf{R}$ ratios in galaxies}

The number ratio $B / R$ of blue to red supergiants is an increasing function of the metallicity $\mathrm{Z}$ in galaxies, typically increasing by a factor of 10 from the SMC to the solar neighbourhood (cf. Humphreys \& McElroy 1984; Langer \& Maeder 1995). Indeed, there is generally no problem for a given set of stellar models to fit the observed $B / R$ at a given $Z$, since $B / R$ at $25-40 M_{\odot}$ typically changes from infinity in case of no mass loss to zero in case of high mass loss. However, it is much more difficult to reproduce the observed decrease of $B / R$ with decreasing Z. As shown by Langer \& Maeder (1995), no assumption in stellar model physics explored so far is able to cope with this trend. All models do the opposite! In general, models with Schwarzschild's criterion can more or less reproduce the observed $B / R$ at $Z=0.02$, but they really fail to do it at low $\mathrm{Z}$, as for example in the SMC. There they predict no or few red supergiants, 
while many are observed. On the other hand, some models with Ledoux criterion correctly reproduce the observed B/R in the SMC (cf. Stothers \& Chin 1992; Brocato \& Castellani 1993), but they generally fail at $\mathrm{Z}=0.01$ or higher (see also Arnett 1991).

For supergiants there is often a stage close to neutrality between a blue and a red location and this is why any change in model assumptions can easily make the star shift from red to blue and vice-versa. Thus the test is a highly sensitive one. In that respect we notice that a restricted scheme for mixing (Ledoux) looks generally better at low $\mathrm{Z}$, while an extended scheme might be better at high $Z$. This suggests some connection of the $B / R$ problem with internal mixing.

\section{The WR stages}

Wolf-Rayet (WR) stars are nowadays considered as bare cores left over from massive stars by mass loss. Recent works have succeeded in explaining the large observed changes of the number ratios $\mathrm{WR} / \mathrm{O}, \mathrm{WC} / \mathrm{WN}$ in galaxies of different metallicities (cf. Maeder \& Meynet 1994). These changes are due to the larger mass loss rates at higher $\mathrm{Z}$. Also, the models were able to explain the observed trend of WC9, WC8 and WC7 to be located in inner regions of the Galaxy. The interesting case of starbursts was also discussed (cf. Maeder \& Conti 1994).

The sequence of Wolf-Rayet stars nicely corresponds to a sequence of more advanced chemical processing becoming visible at the stellar surfaces ( $\mathrm{L}$ means late, E early).

\begin{tabular}{ccccc} 
WNL & WNE & WCL & WCE & WO \\
\hline $\begin{array}{c}\text { CNO } \\
\begin{array}{c}\text { equilibrium } H \\
\text { present }\end{array}\end{array}$ & $\begin{array}{c}\text { CNO } \\
\text { equilibrium }\end{array}$ & $\begin{array}{c}\text { early He } \\
\text { burning }\end{array}$ & $\begin{array}{c}\text { advanced } \\
\text { He-burning }\end{array}$ & $\begin{array}{c}\text { more advanced } \\
O>C\end{array}$
\end{tabular}

The standard models (cf. Schaller et al. 1992) predict an abrupt transition from WN to WC stars, since the He-core is growing and building up a steep chemical discontinuity at its outer edge. Thus the standard models predict almost no $(<1 \%)$ stars with intermediate characteristics of WN and WC stars. However, there are about $4 \%$ of such intermediate stars (Conti \& Massey 1989). It has been shown by Langer (1991) that a mild mixing like that produced by semiconvective diffusion can account for these stars. The existence of WN/WC stars undoubtedly shows that an extra-mixing process is at work, even during the short He-burning phases.

\section{Implications for stellar models}

The various comparisons above tend to favour some additional mixing in stars which leads to more $\mathrm{He}$ and $\mathrm{N}$ brought to the stellar surface during evolution. These abundance excesses generally occur in post-MS evolution (red giants, C-star, supergiants, WR stars); however, in the case of rotating O-stars, the abundance excesses even appear during MS evolution. Indeed, all the above 
facts plead for a profound renewal in the physics of stellar models leading to a new generation of evolutionary grids.

The long standing debate between models with or without overshooting, with or without semiconvection and Ledoux criterion had led to no clear conclusions, some results supporting core overshooting while others support on the contrary restricted cores. The problem has been debated for about 25 years with no generally accepted conclusions.

The effects of mixing due to rotation have also to be considered. This is the case in recent works by Zahn (1992), where the effects of shears are found to be the most important ones. The horizontal shear generates a turbulence, which tends to limit the size of the horizontal shear and to generate a law of angular rotation velocity $\Omega=\Omega(r)$, thus 1-D models may still be meaningful. Secondly, this horizontal turbulence strongly limits the effect of meridional circulation, so that the main mixing effect results from the vertical shear $\frac{\partial \Omega}{\partial r}$. The vertical mixing occurs when $\frac{\partial \Omega}{\partial r}$ is larger than some critical value defined by Richardson's criterion. A new, more consistent form of this criterion has recently been given (Maeder 1995). For sufficiently high $\frac{\partial \Omega}{\partial r}$ the mixing effect can be treated as a diffusion with a coefficient

$$
D_{\text {shear }} \simeq \frac{2}{5} K\left(\frac{\Omega}{N} \frac{\partial \ln \Omega}{\partial \ell n r}\right)^{2}
$$

Mixing thus grows with rotation, where $K$ is the radiative diffusivity $K=\frac{4 a c T^{3}}{3 \kappa \rho^{2} C p}$ and $N$ the Brunt-Väisälä frequency. At the same time a discussion of semiconvection and rotation (Maeder 1995) indicates that semiconvection can only exist for shears $\frac{\partial \Omega}{\partial r}$ smaller than that given by Richardson's criterion. Thus the following picture emerges:

Low initial $\Omega$ and low $\partial \Omega / \partial r$ during evolution

- Ledoux criterion, restricted convection with a mild semiconvective diffusion given by

$$
D_{S C}=\frac{1}{4} K \frac{\left(\nabla_{r a d}-\nabla_{a d}\right)}{\left(\nabla_{a d}-\nabla+\frac{\Gamma+1}{\Gamma} \frac{\varphi}{\delta} \nabla_{\mu}\right)}
$$

where $\nabla_{r a d}$ and $\nabla_{a d}$ are the radiative and adiabatic gradients respectively, $\Gamma$ the Peclet number $(\lesssim 0.1), \varphi$ and $\delta$ thermodynamic coefficients and $\nabla_{\mu}=\left(\frac{\partial \ln \mu}{\partial \ln P}\right)_{P, T}$. High initial $\Omega$ and high $\partial \Omega / \partial r$ during evolution

- Core extended by the effect of $D_{\text {shear }}$ and surrounded by a region of mild mixing, depending on the degree of shear. To some extent the strong shear mixing at the edge of the core may mimic some effects of overshooting. To a first order, in the case of no magnetic coupling between the star and the matter lost in stellar winds, Zahn's results (1992) indicate a local conservation of the angular momentum. Thus the degree of shear $\partial \Omega / \partial r$ during evolution directly results from the value of the initial angular velocity $\Omega$.

Thus the above scheme suggests a change (growth) of the diffusion coefficient with the value of angular velocity. This influences the distribution of elements inside the stars and thus one has

$$
\text { TRACK }=\text { function }(M, Z, \Omega)
$$


It is likely that for given mass $M$ and metallicity $Z$ one no longer has a single evolutionary track (and related results), but a family of tracks depending on $\Omega$. The steep growth of $K$ with stellar mass suggests that such effects will be larger for more massive stars. The observed distributions of stars in the HR diagram and of surface abundances will result from a mixture of tracks of different rotation velocities. Such a mixture of tracks might also well explain the absence of the blue Hertzsprung gap (cf. $\S 7$ ).

On the whole we conclude that the collection of lingering discrepancies shown here demands a new generation of stellar evolutionary models, in which more internal mixing is present. The account for rotational effect is a good line to be explored.

\section{References}

Alongi, M., Bertelli, G., Bressan, A., Chiosi, C., Fagotto, F., et al. 1993, A\&AS, 97,851

Arnett, D. 1991, ApJ, 383, 295

Bertelli, G., Bressan, A., Chiosi, C., Fagotto, F., \& Nasi, E. 1994, A\&AS, 106, 275

Brocato, E., \& Castellani, V. 1993, ApJ, 410, 99

Brocato, E., Castellani, V., \& Piersimoni, A.M. 1994, A\&A, 290, 59

Charbonnel, C. 1994, A\&A, 282, 811

Chiosi, C., Bertelli, G., \& Bressan, A. 1992, ARA\&A, 30, 235

Chiosi, C., Nasi, E., \& Sreenivasan, S.R. 1978, A\&A, 63, 103

Claret, A., \& Gimenez, A. 1992, A\&AS, 96, 255

Conti, P., \& Massey, P. 1989, ApJ, 337, 251

Fitzpatrick, E.L., \& Bohannan, B. 1993, ApJ, 404, 734

Fitzpatrick, E.L., \& Garmany, C.D. 1990, ApJ, 363, 119

Fransson, C., Cassatella, A., Gilmozzi, R., Kirshner, R.P., Panagia, N., et al. 1989, ApJ, 336, 429

Gies, D.R., \& Lambert, D.L. 1992, ApJ, 387, 673

Gilroy, K.K., \& Brown, J.A. 1991, ApJ, 371, 578

Herrero, A., Kudritzki, R.P., Vilchez, J.M., Kunze, D., Butler, K., \& Haser, S. 1992, A\&A, 261, 209

Howarth, I.D., \& Prinja, R.K. 1989, ApJS, 69, 527

Humphreys, R.M., \& McElroy, D.B. 1984, ApJ, 284, 565

Iben, I., \& Tuggle, R.S. 1975, ApJ, 197, 39

Kudritzki, R.P., Hummer, D.G., Pauldrach, A.W.A., Puls, J., Najarro, F., \& Imhoff, J. 1992, A\&A, 257, 655

Lamers, H.J.G.L.M., \& Leitherer, C. 1993, ApJ, 412, 771

Langer, N. 1991, A\&A, 248, 531

Langer, N., \& Maeder A. 1995, A\&A, in press

Lattanzio, J.C. 1991, ApJS, 76, 215 
Lattanzio, J.C., Vallenari, A., Bertelli, G., \& Chiosi, C. 1991, A\&A, 250, 340

Lennon, D.J. 1994, Space Sci. Rev., 66, 127

Luck, R.E., \& Lambert, D.L. 1985, ApJ, 298, 782

Maeder, A. 1987, A\&A, 178, 159

Maeder, A. 1991, QJRAS, 32, 217

Maeder, A. 1995, A\&A, in press

Maeder, A., \& Conti, P. 1994, ARA\&A, 32, 227

Maeder, A., \& Meynet, G. 1989, A\&A, 210, 155

Maeder, A., \& Meynet, G. 1994, A\&A, 287, 803

Meylan, G., \& Maeder, A. 1982, A\&A, 108, 148

Meynet, G., Maeder, A., Schaller, G., Schaerer, D., \& Charbonnel, C. 1994, A\&A, 281, 638

Meynet, G., Mermilliod, J.C., \& Maeder, A. 1993, A\&AS, 98, 477

Mowlawi, N., \& Forestini, M. 1994, A\&A, 282, 843

Nasi, E., \& Forieri, C. 1990, Ap\&SS, 166, 229

Schaerer, D. 1995, Thesis, Univ. Geneva

Schaller, G., Schaerer, D., Meynet, G., \& Maeder, A. 1992, A\&AS, 96, 269

Stothers, R.B., \& Chin, C.W. 1977, ApJ, 211, 189

Stothers, R.B., \& Chin, C.W. 1991, ApJ, 374, 288

Stothers, R.B., \& Chin, C.W. 1992, ApJ, 390, 136

Sweigart, A.V., Greggio, L., \& Renzini, A. 1989, ApJS, 229, 624

Tassoul, J.L. 1978, Theory of rotating stars, Princeton Series in Astroph., p. 3

Tuchman, J., \& Wheeler, J.C. 1989, ApJ, 344, 835

Tuchman, J., \& Wheeler, J.C. 1990, ApJ, 363, 255

VandenBerg, Don A. 1992, ApJ, 391, 685

van Genderen, A.M. 1978, A\&A, 65, 147

Vassiliadis, E., \& Wood, P.R. 1993, ApJ, 413, 641

Venn, K.A. 1993, ApJ, 414, 316

Walborn, N.R. 1976, ApJ, 205, 419

Walborn, N.R. 1988, in Atmospheric Diagnostics of Stellar Evolution, IAU Coll. 108, Ed. K. Nomoto, Springer Verlag, Berlin, p. 70

Zahn, J.P. 1992, A\&A, 265, 115

Zahn, J.P. 1994, Space Sci. Rev., 66, 285

\section{Discussion}

Roxburgh: First, I should stress that the initial magnetic field is also an independent parameter which along with mass, composition and rotation determine the evolution of stars. Next, I wish to stress that the use of the mixing length model to model stellar convective zones is a substantial source of error - since it is not a theory but a model with tunable parameters - and we do not know how to tune the parameters except by either fitting the Sun and applying the 
same value over a wide range of surface parameters - or by adjusting the mixing length to fit each individual observation. But there is at least some hope that numerical simulations can give us better insight into both surface convective zones and convective overshoot in the central regions.

Evans: I might add to your list of problems which the inclusion of rotation may solve. At present up to half the binary Cepheids cannot be fit with isochrones (without rotation). These are binaries with orbital periods longer than a year, so single star evolution should be appropriate.

Maeder: You are quite right, the fact that some binaries, Cepheids or not, do not fit the same isochrone was long recognised as a problem. This is really a point which deserves close attention in future.

Turner: What has changed in recent years to make the treatment of rotation in stellar evolutionary models more tractable (in comparison, say, with your published results of about 10 years ago)?

Maeder: In the previous work of 1987 , it was considered that the horizontal turbulence is the main source of vertical diffusion of chemical elements. Now, more recent works by Zahn (1992) show that the mixing effects of the vertical shears are even more important and that they add, to the first order approximation, their effects to those of horizontal turbulence.

Nemec: For many years there has been a problem understanding why in some globular clusters there are no double-mode RR Lyrae (RRd) stars (e.g., $\omega$ Cen, M5) whereas in other clusters there are many (e.g., IC4499, M15). Faster rotation in $\omega$ Cen and M5 has been advocated as a possible reason for this. Can you comment on this?

Maeder: The interpretation you are mentioning implies that there would be systematic differences in the distributions of rotational velocities (both the initial ones and those in RR Lyrae) for stars in globular clusters. At present there are no observations which permit one to decide on these matters. 